\title{
Model Reduction for linear systems and linear time-delay systems from input/output data
}

\author{
Giordano Scarciotti and Alessandro Astolfi
}

\begin{abstract}
An algorithm for the estimation of the moments of linear single-input, single-output (SISO) systems and linear time-delay SISO systems from input/output data is proposed. It is proved that the estimate converges to the moments of the system. The estimate is exploited to construct a family of reduced order models. These models asymptotically match the moments of the unknown system to be reduced. Conditions to enforce additional properties, e.g. matching with prescribed eigenvalues or matching with prescribed zeros, upon the reduced order model are provided and discussed. The computational complexity of the algorithm is analyzed and the use of the algorithm is illustrated by a benchmark example.
\end{abstract}

\section{INTRODUCTION}

Most of today's technology is based on the availability of some kind of mathematical model of the physical object which has to be built, analyzed or controlled. The advancement of the computational power in the last forty years has been followed by the increasing complexity of these mathematical descriptions which have maintained the computational needs at the top or over the available possibilities [1]. Thus, the need to determine less complex yet meaningful description (in a sense to be specified) is a problem which remains central to the systems and control community. This problem, called model reduction, consists in finding a simplified mathematical model which maintains some key properties of the original model. In the linear framework, in which the concept of complexity has been understood as the dimension of the state of the system, several techniques have been developed. Among these techniques there are some based on the singular value decomposition, see e.g. [2], [3], [4] which make use of Hankel operators and [5], [6], [7], [8] which make use of balanced realizations, and some based on the Krylov projection matrices, see e.g. [9], [10], [11], [12], [13], [14], [15], also called moment matching methods. For additional detail on the model reduction techniques we refer to the monograph [16] and references therein. One of the drawbacks of the techniques based on moment matching is the difficulty in enforcing or preserving important properties of the system to be reduced. However, following the ideas in [17] and [18], this problem has been alleviated. Moreover, the new interpretation given in those papers led to further

G. Scarciotti is with the Dept. of Electrical and Electronic Engineering, Imperial College London, London, SW7 2AZ, UK, E-mail: gs3610@ic.ac.uk.

A. Astolfi is with the Dept. of Electrical and Electronic Engineering, Imperial College London, London, SW7 2AZ, UK and DICII, University of Rome "Tor Vergata", Via del Politecnico 1, 00133 Rome, Italy, Email: a.astolfi@ic.ac.uk. This work is partially supported by the EPSRC Programme Grant "Control For Energy and Sustainability" EP/G066477. developments in the model reduction field, such as the extension of the model reduction theory to linear and nonlinear time-delay systems [19], [20], see also [21], [22], [23], [24]. All these methods assume the knowledge of a representation (e.g. the matrices $A, B, C, D$ ) of the system to be reduced. This is the case, for example, when the model results from the spatial discretization of a distributed parameter system, as in some of the examples in [16]. However, in practice a model of the system to be reduced is not always available. In this paper, inspired by the learning algorithm given in [25] to solve a model-free adaptive dynamic programming problem (see also the references therein, e.g. [26], [27]), we propose an on-line algorithm for the model reduction of linear systems and linear time-delay systems from data. Collecting, at a given sequence of time instants $t_{k}$, timesnapshots (which resemble the ones used to compute a proper orthogonal decomposition (POD), see e.g. [28], [29], [30], [31]) of the input and output of the system, an algorithm is devised to define a family of reduced order models (in the framework introduced in [18]) at each instant of the iteration $t_{k}$. The reduced order model asymptotically matches the moments of the unknown system to be reduced. This algorithm has several advantages with respect to an identification plus reduction technique: there is no need to identify the system, which is expensive both in terms of computational power and storage memory; since the reduced order model matches the moments of the unknown system, it is not just the result of a low-order identification but it actually retains some properties of the larger system; finally, since the technique proposed in [18] can be applied to nonlinear systems, the algorithm developed in this paper has the potential to be exploited to establish a theory of model reduction from data for nonlinear systems.

The rest of the paper is organized as follows. In Section II we recall the definition of moment and the model reduction techniques developed in [18]. In Section III we give a preliminary analysis to compute on-line estimates of the moments of a system assuming the knowledge of the matrices $A, B, C$ : firstly by means of time-snapshots of the input and the state of the system and subsequently, in a more realistic scenario, by means of time-snapshots of the input and the output. In Section IV these on-line estimates are replaced by two approximations which converge asymptotically to the moments of the system. A discussion on the computational complexity associated to the evaluation of these approximations is presented and a recursive leastsquare estimation is given. A moment estimation algorithm is provided and the convergence properties of the algorithm 
are formally proved. In Sections $\mathrm{V}$ and VI we give a family of reduced order models for linear systems and linear time-delay systems, respectively. In Section VII we discuss how several properties, such as matching with prescribed eigenvalues or zeros, can be enforced in the present scenario. In Section VIII an application of the method proposed in the paper is presented using a benchmark system of order $n=1006$ [16], [32]. In particular it is shown how the converging reduced order model approximates increasingly better the system as the approximation of the moments is improved. Finally Section IX contains some concluding remarks and future directions of investigation.

Notation. We use standard notation. $\mathbb{R}_{\geq 0}$ denotes the set of non-negative real numbers; $\mathbb{C}_{<0}$ denotes the set of complex numbers with negative real part; $\mathbb{C}_{0}$ denotes the set of complex numbers with zero real part. The symbol $I$ denotes the identity matrix and $\sigma(A)$ denotes the spectrum of the matrix $A \in \mathbb{R}^{n \times n}$. The symbol $\otimes$ indicates the Kronecker product and $\|A\|$ indicates the induced Euclidean matrix norm. The vectorization of a matrix $A \in \mathbb{R}^{n \times m}$, denoted by $\operatorname{vec}(A)$, is the $n m \times 1$ vector obtained by stacking the columns of the matrix $A$ one on top of the other, namely $\operatorname{vec}(A)=\left[a_{1}^{\top}, a_{2}^{\top}, \ldots, a_{m}^{\top}\right]^{\top}$, where $a_{i} \in \mathbb{R}^{n}$ are the column of $A$ and the superscript $\top$ denotes the transpose. Let $\bar{s} \in \mathbb{C}$ and $A(s) \in \mathbb{C}^{n \times n}$. Then $\bar{s} \notin \sigma(A(s))$ means that $\operatorname{det}(\bar{s} I-A(\bar{s})) \neq 0 . \sigma(A(s)) \subset \mathbb{C}_{<0}$ means that for all $\bar{s}$ such that $\operatorname{det}(\bar{s} I-A(\bar{s}))=0, \bar{s} \in \mathbb{C}_{<0}$.

\section{MOdEL REDUCTION BY MOMENT MATCHING - RECALLED}

To render the paper self-contained in this section we recall the notion of moment for linear systems as presented in [18]. Consider a linear, single-input, single-output, continuoustime, system described by the equations

$$
\dot{x}=A x+B u, \quad y=C x,
$$

with $x(t) \in \mathbb{R}^{n}, u(t) \in \mathbb{R}, y(t) \in \mathbb{R}, A \in \mathbb{R}^{n \times n}, B \in$ $\mathbb{R}^{n \times 1}$ and $C \in \mathbb{R}^{1 \times n}$. Let $W(s)=C(s I-A)^{-1} B$ be the associated transfer function and assume that (1) is minimal, i.e. controllable and observable.

Definition 1: Let $s_{i} \in \mathbb{C}$, with $s_{i} \notin \sigma(A)$. The 0-moment of system (1) at $s_{i}$ is the complex number $\eta_{0}\left(s_{i}\right)=C\left(s_{i} I-\right.$ $A)^{-1} B$. The $k$-moment of system (1) at $s_{i}$ is the complex number

$$
\eta_{k}\left(s_{i}\right)=\frac{(-1)^{k}}{k !}\left[\frac{d^{k}}{d s^{k}}\left(C(s I-A)^{-1} B\right)\right]_{s=s_{i}},
$$

with $k \geq 1$ integer.

In [18] (see also [33] and [14]), a characterization of the moments of system (1) has been given in terms of the solution of a Sylvester equation as follows.

Lemma 1: [18] Consider system (1), $s_{i} \in \mathbb{C}$ and suppose $s_{i} \notin \sigma(A)$, for all $i=1, \ldots, \eta$. There exists a one-to-one relation between the moments $\eta_{0}\left(s_{1}\right), \ldots, \eta_{k_{1}-1}\left(s_{1}\right), \ldots$, $\eta_{0}\left(s_{\eta}\right), \ldots, \eta_{k_{\eta}-1}\left(s_{\eta}\right)$ and the matrix $C \Pi$, where $\Pi$ is the unique solution of the Sylvester equation

$$
A \Pi+B L=\Pi S,
$$

with $S \in \mathbb{R}^{\nu \times \nu}$ any non-derogatory matrix with characteristic polynomial

$$
p(s)=\prod_{i=1}^{\eta}\left(s-s_{i}\right)^{k_{i}}
$$

where $\nu=\sum_{i=1}^{\eta} k_{i}$, and $L$ is such that the pair $(L, S)$ is observable.

In [18] it has also been noted that the moments of system (1) are in one-to-one relation with the well-defined steady-state response of the output of the interconnection between a signal generator with dynamic matrix $S$ and output matrix $L$ (with the properties described in Lemma 1) and system (1). This interpretation of the notion of moment relies upon the center manifold theory, it has the advantage that it can be extended to nonlinear systems and it is of particular interest for the aims of this paper.

Theorem 1: [18] Consider system (1), $s_{i} \in \mathbb{C}$ and suppose $s_{i} \notin \sigma(A)$, for all $i=1, \ldots, \eta$, and $\sigma(A) \in \mathbb{C}_{<0}$. Let $S \in \mathbb{R}^{\nu \times \nu}$ be any non-derogatory matrix with characteristic polynomial (3). Consider the interconnection of system (1) with the system

$$
\dot{\omega}=S \omega, \quad u=L \omega,
$$

with $L$ and $\omega(0)$ such that the triple $(L, S, \omega(0))$ is minimal. Then there exists a one-to-one relation between the moments $\eta_{0}\left(s_{1}\right), \ldots, \eta_{k_{1}-1}\left(s_{1}\right), \ldots, \eta_{0}\left(s_{\eta}\right), \ldots, \eta_{k_{\eta}-1}\left(s_{\eta}\right)$ and the steady-state response of the output $y$ of such interconnected system.

Finally, as shown in [18], the family of systems

$$
\dot{\xi}=(S-G L) \xi+G u, \quad \psi=C \Pi \xi,
$$

with $G$ any matrix such that $\sigma(S) \cap \sigma(S-G L)=\emptyset$, contains all the models of dimension $\nu$ interpolating the moments of system (1) at the eigenvalues of the matrix $S$. Hence, we say that system (5) is a model of (1) at $S$.

\section{A PRELIMINARY ANALYSIS}

In this and the following section we give an algorithm to determine the moments of a linear system described by the equation (1) without assuming any knowledge on the matrices $A, B$ and $C$. The aim of this section is to provide a preliminary analysis to be used for the development of an estimation algorithm. To this end we make the following assumptions.

Assumption 1: The input $u$ of system (1) is described by the equations

$$
\dot{\omega}=S \omega, \quad u=L \omega,
$$

with $S$ such that $\sigma(S) \in \mathbb{C}_{0}$. In addition, assume that the triple $(L, S, \omega(0))$ is minimal, i.e. controllable and observable.

Assumption 2: System (1) is asymptotically stable, i.e. $\sigma(A) \in \mathbb{C}_{<0}$, and minimal.

Assumption 1 has a series of implications. The hypothesis on the eigenvalues of $S$ in reasonable since the contribution 
of the negative eigenvalues of $S$ to the response of the system decays to zero. The minimality of the triple $(L, S, \omega(0))$, which in turn implies the observability of the pair $(L, S)$, guarantees that all the modes of $S$ are present in the signals $\omega$ and $u$ and it can be seen as a condition of persistence of excitation of order $\nu$, see [34]. Note that the choice of the particular structure (6) for the input $u$ is limiting in applications in which the input cannot be arbitrarily chosen. We generalize the class of input in Section IV. Note that the two assumptions imply that $\sigma(A) \cap \sigma(S)=\emptyset$, which in turn implies that equation (2) has a unique solution or, equivalently, that the steady-state in Theorem 1 is well defined and system (1) has a global invariant manifold described by $\mathcal{M}=\left\{(x, \omega) \in \mathbb{R}^{n+\nu}: x=\Pi \omega\right\}$.

We now recall an expression of $x$ in terms of the matrix $\Pi$ that has been derived in the proof of Theorem 1 in [18]. Exploiting the manifold $\mathcal{M}$, it has been shown that the equation

$$
x(t)=\Pi \omega(t)+e^{A t}(x(0)-\Pi \omega(0))
$$

holds. This can be rewritten as

$$
\Pi \omega(t)-e^{A t} \Pi \omega(0)=x(t)-e^{A t} x(0) .
$$

Using the vectorization operator and the Kronecker product on equation (8) yields

$$
\operatorname{vec}(\Pi \omega(t))-\operatorname{vec}\left(e^{A t} \Pi \omega(0)\right)=\operatorname{vec}\left(x(t)-e^{A t} x(0)\right),
$$

and

$$
\left(\omega(t)^{\top} \otimes I-\omega(0)^{\top} \otimes e^{A t}\right) \operatorname{vec}(\Pi)=\operatorname{vec}\left(x(t)-e^{A t} x(0)\right) .
$$

Let the time-snapshots $Q_{k} \in \mathbb{R}^{n p \times n \nu}$ and $\chi_{k} \in \mathbb{R}^{n p}$ be defined as

$$
Q_{k}=\left[\begin{array}{c}
\omega\left(t_{k-p+1}\right)^{\top} \otimes I-\omega(0)^{\top} \otimes e^{A t_{k-p+1}} \\
\vdots \\
\omega\left(t_{k-1}\right)^{\top} \otimes I-\omega(0)^{\top} \otimes e^{A t_{k-1}} \\
\omega\left(t_{k}\right)^{\top} \otimes I-\omega(0)^{\top} \otimes e^{A t_{k}}
\end{array}\right],
$$

and

$$
\chi_{k}=\left[\begin{array}{c}
x\left(t_{k-p+1}\right)-e^{A t_{k-p+1}} x(0) \\
\vdots \\
x\left(t_{k-1}\right)-e^{A t_{k-1}} x(0) \\
x\left(t_{k}\right)-e^{A t_{k}} x(0)
\end{array}\right]
$$

respectively, where $0 \leq t_{0}<t_{1}<\cdots<t_{k-p}<\cdots<$ $t_{k}<\cdots<t_{q}$, with $p>0$ and $q \geq p$. Let $T_{k}^{p}=$ $\left\{t_{k-p+1}, \ldots, t_{k-1}, t_{k}\right\}$ and $\Pi_{k}$ be an on-line estimate of the matrix $\Pi$ computed at $T_{k}^{p}$, namely computed at the time $t_{k}$ using the last $p$ instants of time $t_{i}$. Computing equation (9) at all elements of $T_{k}^{p}$ yields

$$
Q_{k} \operatorname{vec}\left(\Pi_{k}\right)=\chi_{k} .
$$

If the matrix $Q_{k}$ has full rank, we can compute $\Pi_{k}$ from the last equation as

$$
\operatorname{vec}\left(\Pi_{k}\right)=\left(Q_{k}^{\top} Q_{k}\right)^{-1} Q_{k}^{\top} \chi_{k}
$$

Note that the selection of the set $T_{k}^{p}$ can affect the quality of the data and the rank of the matrix $Q_{k}$. For this reason we introduce the following technical assumption.

Assumption 3: The elements of $T_{k}^{\nu}$ are such that $\operatorname{rank}\left(\left[\begin{array}{lll}\omega\left(t_{k-\nu+1}\right) & \ldots & \omega\left(t_{k}\right)\end{array}\right]\right)=\nu$ for all $k$.

Lemma 2: Suppose Assumptions 1, 2 and 3 hold. If $p=$ $\nu, Q_{k}$ is full rank.

Proof: Let $x(0) \neq \Pi \omega(0)$ and consider the $i$-block element of the matrix $Q_{k}$, namely

$$
\omega\left(t_{i}\right)^{\top} \otimes I-\omega(0)^{\top} \otimes e^{A t_{i}} .
$$

Note that the properties of the Kronecker product yield

$$
\begin{aligned}
& \omega\left(t_{i}\right)^{\top} \otimes I-\omega(0)^{\top} \otimes e^{A t_{i}}= \\
& =\omega(0)^{\top} e^{S^{\top} t_{i}} \otimes I I-\omega(0)^{\top} I \otimes I e^{A t_{i}}= \\
& =\left(\omega(0)^{\top} \otimes I\right)\left(e^{S^{\top} t_{i}} \otimes I\right)-\left(\omega(0)^{\top} \otimes I\right)\left(I \otimes e^{A t_{i}}\right)= \\
& =\left(\omega(0)^{\top} \otimes I\right)\left(e^{S^{\top} t_{i}} \otimes I-I \otimes e^{A t_{i}}\right) .
\end{aligned}
$$

Since $\sigma(A) \in \mathbb{C}_{<0}$ and $\sigma(S) \in \mathbb{C}_{0}$, the controllability of $(S, \omega(0))$ implies that the $i$-block element of the matrix $Q_{k}$ is a $n \times n \nu$ matrix of rank $n$. Assumption 3 implies that $\nu$ of these blocks are linearly independent for any $t_{i}>0$. As a result $Q_{k}$ is a square full rank matrix.

Let $x(0)=\Pi \omega(0)$. Since equation (9) reduces to

$$
\left(\omega(t)^{\top} \otimes I\right) \operatorname{vec}(\Pi)=\operatorname{vec}(x(t)),
$$

the $i$-block element of the matrix $Q_{k}$ is

$$
\left(\omega(0)^{\top} \otimes I\right)\left(e^{S^{\top} t_{i}} \otimes I\right) .
$$

Assumption 3 and the controllability of $(S, \omega(0))$ guarantee that $\nu$ of these blocks are linearly independent for any $t_{i} \geq 0$, from which the claim follows.

Remark 1: Note that $p$ cannot be less than $\nu$. In fact, (11) consists in $n \nu$ (the number of entries of $\Pi_{k}$ ) linear equations.

Remark 2: Since real data are affected by noise the assumptions of Lemma 2 may not hold. In this case $p$ can be taken larger than $n \nu$ and, as well-known from linear algebra and remarked in [25] and [34], the solution of equation (11) is the least squares solution of (10).

The discussion carried out so far has the drawback that requires the information on the state of the system. In practice, this is usually not the case and only the output $y$ may be available. In this case, define the time-snapshots $R_{k} \in \mathbb{R}^{w \times n \nu}$ and $\Upsilon_{k} \in \mathbb{R}^{w}$ as

$$
R_{k}=\left[\begin{array}{c}
\left(\omega(0)^{\top} \otimes C\right)\left(e^{S^{\top} t_{k-w+1}} \otimes I-I \otimes e^{A t_{k-w+1}}\right) \\
\vdots \\
\left(\omega(0)^{\top} \otimes C\right)\left(e^{S^{\top} t_{k-1}} \otimes I-I \otimes e^{A t_{k-1}}\right) \\
\left(\omega(0)^{\top} \otimes C\right)\left(e^{S^{\top} t_{k}} \otimes I-I \otimes e^{A t_{k}}\right)
\end{array}\right],
$$

and

$$
\Upsilon_{k}=\left[\begin{array}{c}
y\left(t_{k-w+1}\right)-C e^{A t_{k-w+1}} x(0) \\
\vdots \\
y\left(t_{k-1}\right)-C e^{A t_{k-1}} x(0) \\
y\left(t_{k}\right)-C e^{A t_{k}} x(0)
\end{array}\right]
$$


The same steps used to obtain equation (11) yield the on-line estimate

$$
\operatorname{vec}\left(\Pi_{k}\right)=\left(R_{k}^{\top} R_{k}\right)^{-1} R_{k}^{\top} \Upsilon_{k}
$$

Lemma 3: Suppose Assumptions 1, 2 and 3 hold. If $w=$ $n \nu, R_{k}$ is full rank.

Proof: The proof is similar to the one of Lemma 2. Note that this time also the minimality of $(A, B, C)$ is needed.

\section{ON-LINE MODEL REDUCTION FROM DATA}

Equation (11) contains terms which depend upon the matrix $A$ and the initial states $x(0)$ and $\omega(0)$. However, we note that given the stability hypothesis on the system, these terms are exponentially decaying functions. Thus, let $\widetilde{\Pi}$ be such that

$$
x(t)=\widetilde{\Pi} \omega(t)
$$

and define the time-snapshots $\widetilde{Q}_{k} \in \mathbb{R}^{n p \times n \nu}$ and $\widetilde{\chi}_{k} \in \mathbb{R}^{n p}$ as

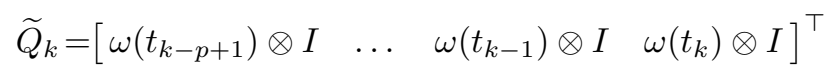

and

$$
\tilde{\chi}_{k}=\left[\begin{array}{lll}
x\left(t_{k-p+1}\right)^{\top} \quad \ldots & x\left(t_{k-1}\right)^{\top} x\left(t_{k}\right)^{\top}
\end{array}\right]^{\top}
$$

Then

$$
\operatorname{vec}\left(\widetilde{\Pi}_{k}\right)=\left(\widetilde{Q}_{k}^{\top} \widetilde{Q}_{k}\right)^{-1} \widetilde{Q}_{k}^{\top} \widetilde{\chi}_{k}
$$

is an approximation of the on-line estimate $\Pi_{k}$.

Remark 3: Equation (13) may remind the POD of the collection $\left\{x\left(t_{i}\right)\right\}$. However, the two concepts are quite different. In fact, the POD of $\left\{x\left(t_{i}\right)\right\}$ is

$$
\left[\begin{array}{lll}
x\left(t_{0}\right) & \ldots & x\left(t_{q}\right)
\end{array}\right]=\underbrace{\left[\begin{array}{lll}
u\left(t_{0}\right) & \ldots & u\left(t_{q}\right)
\end{array}\right]}_{U} \Gamma
$$

with $\Gamma \in \mathbb{R}^{q \times q}$ and $U^{*} U=I$, where the superscript $*$ indicates the complex conjugate transpose. The POD is a decomposition of the entire cloud of data $\left\{x\left(t_{i}\right)\right\}$ along the vectors $u\left(t_{i}\right)$, called principal directions of $\left\{x\left(t_{i}\right)\right\}$ [16]. The dimensions of $\Gamma$ are related to the number of samples, whereas the dimensions of $\Pi$ are related to the ones of the system to be reduced and of the signal generator system. The consequence is that $\Gamma$ is built to describe the entire dynamics of $\left\{x\left(t_{i}\right)\right\}$, whereas $\Pi$ is built to describe the steady-state response of the system to be reduced. The result is that the POD is usually used with Petrov-Galerkin projection for a SVD-based approximation [29], [31], whereas this technique is a moment matching method.

A similar discussion can be carried out for equation (12) that contains also terms which depend upon the matrix $C$. In this case note that equation (7) can be written as

$$
y(t)=C \Pi \omega(t)+\varepsilon(t),
$$

with $\varepsilon(t)=C e^{A t}(x(0)-\Pi \omega(0))$ an exponentially decaying signal. Thus, let $\widehat{C \Pi}$ be such that

$$
y(t)=\widetilde{C \Pi} \omega(t)
$$

and define the time-snapshots $\widetilde{R}_{k} \in \mathbb{R}^{w \times \nu}$ and $\widetilde{\Upsilon}_{k} \in \mathbb{R}^{w}$ as

$$
\widetilde{R}_{k}=\left[\begin{array}{llll}
\omega\left(t_{k-w+1}\right) & \ldots & \omega\left(t_{k-1}\right) & \omega\left(t_{k}\right)
\end{array}\right]^{\top}
$$

and

$$
\widetilde{\Upsilon}_{k}=\left[\begin{array}{llll}
y\left(t_{k-w+1}\right) & \ldots & y\left(t_{k-1}\right) & y\left(t_{k}\right)
\end{array}\right]^{\top} .
$$

Then

$$
\operatorname{vec}\left(\widetilde{C \Pi}_{k}\right)=\left(\widetilde{R}_{k}^{\top} \widetilde{R}_{k}\right)^{-1} \widetilde{R}_{k}^{\top} \widetilde{\Upsilon}_{k},
$$

is an approximation of the on-line estimate $C \Pi_{k}$. Note that if Assumption 3 holds and $w=\nu, \widetilde{R}_{k}$ is full rank.

Remark 4: The matrix $\widetilde{R}_{k}$ is considerably smaller than $R_{k}$ since is not obtained from Kronecker products.

With equation (15) we are not able to retrieve the matrix $\widetilde{\Pi}_{k}$, but only $\widetilde{C \Pi}_{k}$. However, as shown in equation (5), we only need $C \Pi$ to compute the reduced order model, i.e. $\Pi$ is not explicitly required.

Remark 5: The larger contribution to the computational complexity of determining $\widetilde{C \Pi}_{k}$ from equation (15) comes from the inversion of the matrix $\widetilde{R}_{k}^{\top} \widetilde{R}_{k}$. However, we note that recursive algorithms can be implemented. In particular, a combination of the Greville algorithm [35], [36] (to add a new line) and the Cline algorithm [36], [37] (to remove an old line) can be exploited to update recursively the matrix $\left(\widetilde{R}_{k}^{\top} \widetilde{R}_{k}\right)^{-1} \widetilde{R}_{k}^{\top}$. Then the computational complexity of solving equation (15) is $\mathcal{O}(\nu w)$, which with low-noise measurements, i.e. when Assumption 3 holds, becomes $\mathcal{O}\left(\nu^{2}\right)$ (note that $\nu \ll n$ ). In comparison, the Arnoldi or Lanczos procedure for the model reduction by moment matching have a computational complexity of $\mathcal{O}\left(\nu n^{2}\right)$ [16, Section 14.1] (or $\mathcal{O}(\alpha \nu n)$ for a sparse matrix $A$, with $\alpha$ the average number of non-zero elements per row/column of $A$ ). In addition, note that these procedures require a model to be reduced and thus further expensive computation has to be considered for the identification of the original system.

Equation (15) is a classic least-square estimation formula. Adapting the discussion in [34] to the present scenario, in which at each step we acquire a new measure and we discard an old measure, it is easy to derive a recursive least-squares estimation of $\widetilde{C \Pi}_{k}$. To this end, let

$$
\begin{aligned}
& \Phi_{k}=\left(\widetilde{R}_{k}^{\top} \widetilde{R}_{k}\right)^{-1}, \\
& \Psi_{k}=\left(\widetilde{R}_{k-1}^{\top} \widetilde{R}_{k-1}+\omega\left(t_{k}\right) \omega\left(t_{k}\right)^{\top}\right)^{-1} .
\end{aligned}
$$

Then

$$
\begin{aligned}
\widetilde{C \Pi}_{k} & =\widetilde{C \Pi}_{k-1}+\Phi_{k} \omega\left(t_{k}\right)\left(y\left(t_{k}\right)-\omega\left(t_{k}\right)^{\top} \widetilde{C \Pi}_{k-1}\right) \\
& -\Phi_{k} \omega\left(t_{k-w}\right)\left(y\left(t_{k-w}\right)-\omega\left(t_{k-w}\right)^{\top} \widetilde{C \Pi}_{k-1}\right),
\end{aligned}
$$

with

$$
\begin{aligned}
\Phi_{k} & =\Psi_{k}-\Psi_{k} \omega\left(t_{k-w}\right) \times \\
& \times\left(I+\omega\left(t_{k-w}\right)^{\top} \Psi_{k} \omega\left(t_{k-w}\right)\right)^{-1} \omega\left(t_{k-w}\right)^{\top} \Psi_{k}
\end{aligned}
$$

and

$$
\begin{aligned}
\Psi_{k} & =\Phi_{k-1}-\Phi_{k-1} \omega\left(t_{k}\right) \times \\
& \times\left(I+\omega\left(t_{k}\right)^{\top} \Phi_{k-1} \omega\left(t_{k}\right)\right)^{-1} \omega\left(t_{k}\right)^{\top} \Phi_{k-1} .
\end{aligned}
$$


Note that for single-input, single-output systems the two matrix inversions in the definition of $\Phi_{k}$ and $\Psi_{k}$ are two divisions. Equations (16)-(17)-(18) can be used to compute a fast, on-line, estimate of $\widetilde{C \Pi}_{k}$, since the computation complexity of updating (16) is $\mathcal{O}(1)$. Thus, the implementation of equations (16)-(17)-(18) is preferred to equation (15).

The approximations $\widetilde{\Pi}_{k}$ and $\widetilde{C \Pi}_{k}$ can be computed with the following algorithm.

Algorithm 1:

1) Set $k=0$.

2) Let $k=k+1$. Construct the matrices $\widetilde{Q}_{k}, \widetilde{\chi}_{k}$ (or $\widetilde{R}_{k}$, $\widetilde{\Upsilon}_{k}$ ) and repeat Step 2 until the matrix $\widetilde{Q}_{k}$ (or $\widetilde{R}_{k}$ ) is full rank.

3) Solve equation (14) (or (15), or (16)).

4) Repeat Step 2 until

$$
\left\|\widetilde{\Pi}_{k}-\widetilde{\Pi}_{k-1}\right\| \leq \frac{\eta}{t_{k}-t_{k-1}},
$$

or

$$
\left\|\widetilde{C \Pi}_{k}-\widetilde{C \Pi}_{k-1}\right\| \leq \frac{\eta}{t_{k}-t_{k-1}},
$$

with $\eta$ a small strictly positive number which has the role of threshold.

We prove now that $\Pi_{k}$ is exactly $\Pi$, and that $\widetilde{\Pi}_{k}$ and $\widetilde{C \Pi}_{k}$ converge to $\Pi$ and $C \Pi$, respectively.

Assumption 4: There exists a matrix $\bar{\Pi}$ such that $\lim _{k \rightarrow \infty} \widetilde{\Pi}_{k}=\bar{\Pi}$.

Theorem 2: Suppose Assumptions 1, 2, 3 and 4 hold. Let $\Pi$ be the solution of equation (2). Then $\Pi_{k}$ computed by equation (11) or (12) is equal to $\Pi$. Moreover, $\widetilde{\Pi}_{k}$ and $\widetilde{C \Pi}_{k}$ converge to $\Pi$ and $C \Pi$, respectively.

Proof: To simplify the discussion we consider the case of $\Pi_{k}$ from equation (11) and $\widetilde{\Pi}_{k}$ from equation (14), which contains all ingredients necessary for the other case. Let $t_{k}$ be such that $\omega\left(t_{k}\right) \neq 0$ and note that such a $t_{k}$ always exists. The matrix $\Pi_{k}$ defined in equation (11) is such that the equations

$$
x\left(t_{k}\right)=\Pi_{k} \omega\left(t_{k}\right)+e^{A t_{k}}\left(x(0)-\Pi_{k} \omega(0)\right)
$$

and

$$
\left.\dot{x}(t)\right|_{t_{k}}=\Pi_{k} S \omega\left(t_{k}\right)+A e^{A t_{k}}\left(x(0)-\Pi_{k} \omega(0)\right)
$$

hold. Consider the first equation of system (1) computed at $t_{k}$, namely

$$
\left.\dot{x}(t)\right|_{t_{k}}=A x\left(t_{k}\right)+B L \omega\left(t_{k}\right) .
$$

Substituting equation (19) and (20) in equation (21) yields

$$
\begin{aligned}
& \Pi_{k} S \omega\left(t_{k}\right)+A e^{A t_{k}}\left(x(0)-\Pi_{k} \omega(0)\right)= \\
& A\left(\Pi_{k} \omega\left(t_{k}\right)+e^{A t_{k}}(x(0)-\Pi \omega(0))\right)+B L \omega\left(t_{k}\right),
\end{aligned}
$$

from which

$$
A \Pi_{k}+B L=\Pi_{k} S
$$

follows. Since $\sigma(A) \cap \sigma(S)=\emptyset$, this last equation has a unique solution, hence $\Pi_{k}=\Pi$.

The matrix $\widetilde{\Pi}_{k}$ defined in equation (14) is such that

$$
x\left(t_{k}\right)=\widetilde{\Pi}_{k} \omega\left(t_{k}\right) .
$$

Substituting equation (22) and (20) in equation (21) yields

$$
\begin{aligned}
& \Pi_{k} S \omega\left(t_{k}\right)+A e^{A t_{k}}(x(0)-\left.\Pi_{k} \omega(0)\right)= \\
& A \widetilde{\Pi}_{k} \omega\left(t_{k}\right)+B L \omega\left(t_{k}\right)
\end{aligned}
$$

and since $\Pi_{k}=\Pi$,

$$
\left(A \widetilde{\Pi}_{k}+B L-\Pi S\right) \omega\left(t_{k}\right)=A e^{A t_{k}}(x(0)-\Pi \omega(0)),
$$

from which, using equation (2) and Assumption 2,

$$
\left(\widetilde{\Pi}_{k}-\Pi\right) \omega\left(t_{k}\right)=e^{A t_{k}}(x(0)-\Pi \omega(0))
$$

follows. By Assumption 1 there exists a sequence $\left\{t_{k}\right\}$, with $\lim _{k \rightarrow \infty} t_{k}=\infty$, such that for any $t_{i} \in\left\{t_{k}\right\}, \omega\left(t_{i}\right) \neq 0$ and Assumption 3 holds. By Assumption 2

$$
\lim _{k \rightarrow \infty}\left(\widetilde{\Pi}_{k}-\Pi\right) \omega\left(t_{k}\right)=\lim _{k \rightarrow \infty} e^{A t_{k}}(x(0)-\Pi \omega(0))=0 .
$$

and by Assumptions 3 and 4, $\lim _{k \rightarrow \infty}(\bar{\Pi}-\Pi)=0$. It follows that $\widetilde{\Pi}_{k}$ converges asymptotically to $\Pi$.

Remark 6: It is not always possible to arbitrarily select the input of the system to be reduced. For instance the input signal may be composed by several unwanted frequencies. Instead of system (6), consider the input described by the equations

$$
\dot{\omega}=S \omega, \quad u=L \omega+v,
$$

with $v(t) \in \mathbb{R}^{n}$ an unknown signal. In this case equation (7) (for the output $y$ ) is

$y(t)=C \Pi \omega(t)+C e^{A t}(x(0)-\Pi \omega(0))+\int_{0}^{t} e^{A(t-\tau)} B v(\tau) d \tau$,

which can be written as

$$
y(t)=C \Pi \omega(t)+\varepsilon(t)+\mathfrak{v}(t),
$$

with $\mathfrak{v}(t)=\int_{0}^{t} e^{A(t-\tau)} B v(\tau) d \tau$ and $\varepsilon(t)=C e^{A t}(x(0)-$ $\Pi \omega(0))$. One can then apply the filtering techniques explained in [34, Chapter 11]: we filter out $\mathfrak{v}$ from $y$ and $u$ with a band-pass filter and apply the results of the paper to the filtered $y_{f}$ and $u_{f}$.

\section{FAMILIES OF REDUCED ORDER MODELS}

Using the approximations given by Algorithm 1 a reduced order model of system (1) can be defined at each instant of time $t_{k}$.

Definition 2: Consider system (1) and the signal generator (6). Suppose Assumptions 1, 2, 3 and 4 hold. Then the system

$$
\begin{aligned}
& \dot{\xi}=F_{k} \xi+G_{k} u, \\
& \phi=H_{k} \xi,
\end{aligned}
$$

with $\xi(t) \in \mathbb{R}^{\nu}, F_{k} \in \mathbb{R}^{\nu \times \nu}, G_{k} \in \mathbb{R}^{\nu \times 1}, H_{k} \in \mathbb{R}^{1 \times \nu}$, is a model of system (1) at (6) at time $t_{k}$, if there exists a unique solution $P_{k}$ of the equation

$$
F_{k} P_{k}+G_{k} L=P_{k} S
$$


such that

$$
\widetilde{C \Pi}_{k}=H_{k} P_{k},
$$

where $\widetilde{C \Pi}_{k}$ is the solution of (15).

Remark 7: Select $P_{k}=I$, for all $k \geq 0$. If $\sigma\left(F_{k}\right) \cap$ $\sigma(S)=\emptyset$ for all $k \geq 0$, then the model

$$
\begin{aligned}
\dot{\xi} & =\left(S-G_{k} L\right) \xi+G_{k} u, \\
\phi & =\widetilde{C \Pi}_{k} \xi,
\end{aligned}
$$

is a model of system (1) at (6) for all $t_{k}$.

\section{LINEAR TIME-DELAY SYSTEMS}

The results developed so far can be easily extended to linear time-delay systems. In fact, consider a linear, singleinput, single-output, continuous-time, time-delay system described by the equations

$$
\begin{aligned}
& \dot{x}(t)=A_{0} x(t)+\sum_{j=1}^{\mu} A_{j} x\left(t-\tau_{j}\right)+B u\left(t-\tau_{u}\right), \\
& y(t)=C x(t),
\end{aligned}
$$

with $x(t) \in \mathbb{R}^{n}, u(t) \in \mathbb{R}, y(t) \in \mathbb{R}, A_{j} \in \mathbb{R}^{n \times n}$ with $j=0, \ldots, \mu, B \in \mathbb{R}^{n \times 1}, C \in \mathbb{R}^{1 \times n}, \tau_{j} \in \mathbb{R}_{\geq 0}$ with $j=$ $1, \ldots, \mu, \tau_{u} \in \mathbb{R}_{\geq 0}$. Let $\bar{A}(s)=A_{0}+\sum_{j=1}^{\mu} e^{-s \tau_{j}} A_{j}$. As established in [19], if $s_{i} \notin \sigma(\bar{A}(s))$ for all $s_{i} \in \sigma(S)$, the moments of system (27), namely

$$
\begin{aligned}
& \eta_{k}\left(s_{i}\right)= \\
& =\frac{(-1)^{k}}{k !}\left[\frac{d^{k}}{d s^{k}}\left(C\left(s I-A_{0}-\sum_{j=1}^{\mu} e^{-s \tau_{j}} A_{j}\right)^{-1} e^{-s \tau_{u}} B\right)\right]_{s=s_{i}},
\end{aligned}
$$

are in one-to-one relation with the matrix $C \Pi$, where $\Pi$ is the unique solution of the Sylvester-like equation

$$
A_{0} \Pi+\sum_{j=1}^{\mu} A_{j} \Pi e^{-S \tau_{j}}-\Pi S=-B L e^{-S \tau_{u}} .
$$

In [20] it has been shown that if in addition $\sigma(\bar{A}(s)) \subset \mathbb{C}_{<0}$ then

$$
y(t)=C \Pi \omega(t)+\varepsilon(t),
$$

with $\varepsilon(t)=C \mathcal{L}^{-1}\left\{(s I-\bar{A}(s))^{-1}(x(0)-\Pi \omega(0))\right\}$ an exponentially decaying function. Thus, Theorem 2 holds for the linear time-delay system (27) (the proof is a simple exercise).

Definition 3: Consider system (27) and the signal generator (6). Assume $\sigma(\bar{A}(s)) \subset \mathbb{C}_{<0}$, system (27) is minimal and suppose Assumptions 1, 3 and 4 hold. Then the system

$$
\begin{aligned}
& \dot{\xi}(t)=F_{0, k} \xi(t)+\sum_{j=1}^{\rho} F_{j, k} \xi\left(t-\chi_{j}\right)+G_{k} u\left(t-\chi_{u}\right), \\
& \phi(t)=H_{k} \xi(t),
\end{aligned}
$$

with $\xi(t) \in \mathbb{R}^{\nu}, \phi(t) \in \mathbb{R}, F_{j, k} \in \mathbb{R}^{\nu \times \nu}$ for $j=0, \ldots, \rho \geq$ $0, \chi_{j} \in \mathbb{R}_{\geq 0}$ for $j=1, \ldots, \rho, \chi_{u} \in \mathbb{R}_{\geq 0}, G_{k} \in \mathbb{R}^{\nu \times 1}$ and
$H_{k} \in \mathbb{R}^{1 \times \nu}$, is a model of system (27) at (6) at time $t_{k}$, if there exists a unique solution $P_{k}$ of the equation

$$
F_{0, k} P_{k}+\sum_{j=1}^{\rho} F_{j, k} P_{k} e^{-S \chi_{j}}-P_{k} S=-G_{k} L e^{-S \chi_{u}},
$$

such that

$$
\widetilde{C \Pi}_{k}=H_{k} P_{k},
$$

where $\widetilde{C \Pi}_{k}$ is the solution of (15).

Remark 8: Let $\bar{F}_{k}(s)=F_{0, k}-\sum_{j=1}^{\rho} F_{j, k} e^{-s \chi_{j}}$ and select $P_{k}=I$ for all $k \geq 0$. If $\sigma\left(\bar{F}_{k}(s)\right) \cap \sigma(S)=\emptyset$ for all $k \geq 0$, then the model

$$
\begin{aligned}
\dot{\xi}(t)=\left(S-G_{k} L e^{-S \chi_{u}}\right. & \left.-\sum_{j=1}^{\rho} F_{j, k} e^{-S \chi_{j}}\right) \xi(t)+ \\
& +\sum_{j=1}^{\rho} F_{j, k} \xi\left(t-\chi_{j}\right)+G_{k} u\left(t-\chi_{u}\right),
\end{aligned}
$$

$\phi(t)=\widetilde{C \Pi}_{k} \xi(t)$,

is a model of system (27) at (6) for all $t_{k}$.

\section{PROPERTIES OF THE EXPONENTIALLY CONVERGING MODELS}

In [18], [19] the problem of enforcing additional properties and constraints on the reduced order model has been studied. In this section we briefly go through these properties to determine if, and under which conditions, they hold for the models (26) and (32).

\section{A. Matching with prescribed eigenvalues}

Consider system (26) and the problem of determining at every $k$ the matrix $G_{k}$ such that $\sigma\left(F_{k}\right)=\left\{\lambda_{1, k}, \ldots, \lambda_{\nu, k}\right\}$ for some prescribed values $\lambda_{i, k}$. The solution of this problem is well-known and consists in selecting $G_{k}$ such that

$$
\sigma\left(S-G_{k} L\right)=\sigma\left(F_{k}\right) .
$$

This is possible for every $k$ and for all $\lambda_{i, k} \notin \sigma(S)$ and note that $G_{k}$ is independent from the estimate $\widetilde{C \Pi}_{k}$. Note also that by observability of $(L, S), G_{k}$ is unique at every $k$.

\section{B. Matching with interpolation a $2 \nu$ points}

Let $S_{a} \in \mathbb{R}^{\nu \times \nu}$ and $S_{b} \in \mathbb{R}^{\nu \times \nu}$ be two non-derogatory matrices such that $\sigma\left(S_{a}\right) \cap \sigma\left(S_{b}\right)=\emptyset$ and let $L_{a}$ and $L_{b}$ be such that the pairs $\left(L_{a}, S_{a}\right)$ and $\left(L_{b}, S_{b}\right)$ are observable. Let $\widetilde{C \Pi}_{k}=\widetilde{C \Pi}_{a, k}$ be the unique solution of (15) with $S=S_{a}$ and let $\widetilde{C \Pi}_{k}=\widetilde{C \Pi}_{b, k}$ be the unique solution of (15) with $S=S_{b}$. Consider system (32) with $\rho=1$ and the problem of determining $F_{1, k}$ such that system (32) is a model of system (27) at $S_{a}$ and $S_{b}$ at time $t_{k}$. This problem is solved selecting

$$
\begin{aligned}
F_{1, k}=\left(P_{b, k} S_{b}-S_{a} P_{b, k}\right. & \left.+G_{k} L_{a} P_{b, k}-G_{k} L_{b}\right) \times \\
& \times\left(P_{b, k} e^{-S_{b} \chi_{1}}-e^{-S_{a} \chi_{1}} P_{b, k}\right)^{-1},
\end{aligned}
$$




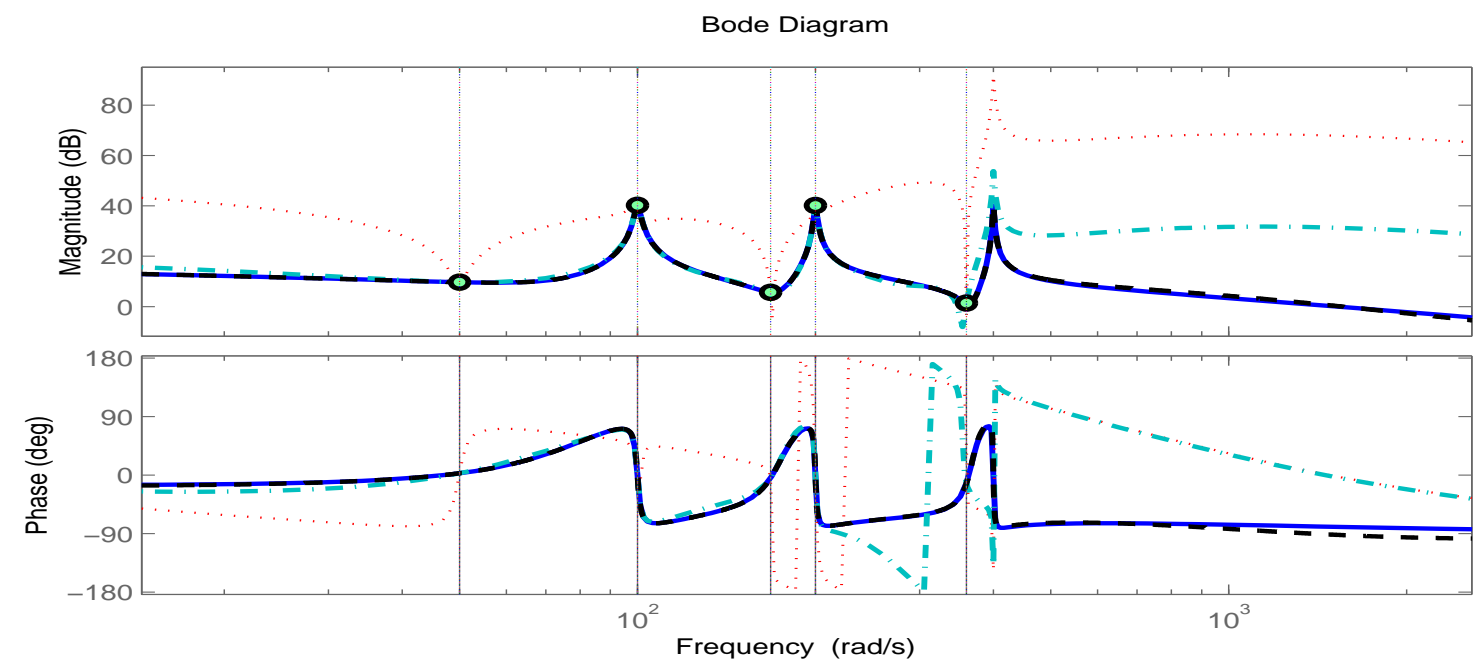

Fig. 1. Bode plot of the system (solid line), of the reduced order model at $t_{k}=90 \mathrm{~s}$ (dotted line), of the reduced order model at $t_{k}=110 \mathrm{~s}$ (dash-dotted line) and of the reduced order model at $t_{k}=140 \mathrm{~s}$ (dashed line). The circles indicate the interpolation points.

and $P_{b, k}$ such that

$$
\widetilde{C \Pi}_{a, k} P_{b, k}=\widetilde{C \Pi}_{b, k} .
$$

This last condition may not be satisfied for all $k$. In fact, it is easy to note that for some $k, \widetilde{C \Pi}_{a, k}$ may be zero even though the asymptotic value is not zero. Note that the observability of the pairs $\left(S_{a}, L_{a}\right)$ and $\left(S_{a}, L_{a}\right)$ guarantees that (34) is solvable for the asymptotic values of $\widetilde{C \Pi}_{a, k}$ and $\widetilde{C \Pi}_{b, k}$. Hence, there exists $\bar{k} \gg 0$ such that for all $k \geq \bar{k}$ equation (34) has a solution.

C. Matching with prescribed relative degree, matching with prescribed zeros, matching with compartmental constraints

These problems can be solved at each $k$ as detailed in [18] if and only if

$$
\operatorname{rank}\left[\begin{array}{c}
s I-S \\
\widetilde{C \Pi_{k}}
\end{array}\right]=n,
$$

for all $s \in \sigma(S)$ at $k$. Even though the asymptotic value of $\widetilde{C \Pi}_{k}$ satisfies this condition there is no guarantee that the condition holds for all $k$. However, if the condition holds for the asymptotic value, there exists $\bar{k} \gg 0$ such that for all $k \geq \bar{k}$ equation (34) has a solution.

\section{APPLICATION TO A SYSTEM OF ORDER $n=1006$}

In this section we apply Algorithm 1 to the example given in [32] (see also [16]). The example is a single-input, singleoutput system of order $n=1006$. It is a theoretical test which has a Bode plot with three peaks. The state space matrices of system (1) are given by $A=\operatorname{diag}\left(A_{1}, A_{2}, A_{3}, A_{4}\right)$, with

$$
\begin{gathered}
A_{1}=\left[\begin{array}{cc}
-1 & 100 \\
-100 & -1
\end{array}\right], \quad A_{2}=\left[\begin{array}{cc}
-1 & 200 \\
-200 & -1
\end{array}\right], \\
A_{3}=\left[\begin{array}{cc}
-1 & 400 \\
-400 & -1
\end{array}\right], \quad A_{4}=\operatorname{diag}(-1,-2, \ldots,-1000),
\end{gathered}
$$

$$
\text { and } B^{\top}=C=[\underbrace{10 \ldots 10}_{6 \text { times }} \underbrace{1 \ldots 1}_{1000 \text { times }}] \text {. }
$$

The matrices of the signal generator (6) has been selected as $S=\operatorname{diag}\left(0, S_{1}, S_{2}, S_{3}, S_{4}, S_{5}\right)$, with $S_{1}=A_{1}+I, S_{2}=$ $1.68 S_{1}, S_{3}=2 S_{1}, S_{4}=3.6 S_{1}$ and

$$
S_{5}=\left[\begin{array}{cc}
\frac{1}{2} S_{1} & I \\
0 & \frac{1}{2} S_{1}
\end{array}\right],
$$

to interpolate the moments at 0 and close to the three frequency peaks.

A reduced order model (26) at time $t_{k}$ has been constructed assigning the eigenvalues of $F_{k}$. Fig. 1 shows the Bode plot of the system (solid line), of the reduced order model at $t_{k}=90 \mathrm{~s}$ (dotted line), of the reduced order model at $t_{k}=110 \mathrm{~s}$ (dash-dotted line) and of the reduced order model at $t_{k}=140 \mathrm{~s}$ (dashed line). Note that the frequencies of interest, indicated with circles, are interpolated already at $t_{k}=90 \mathrm{~s}$. Fig. 2 shows the respective absolute errors between the Bode plot of the system to be reduced and the Bode plot of the reduced order model at $t_{k}=90 \mathrm{~s}$ (dotted line), of the reduced order model at $t_{k}=110 \mathrm{~s}$ (dash-dotted line) and of the reduced order model at $t_{k}=140 \mathrm{~s}$ (dashed line). We see that the error between the reduced order model and the system decreases as $t_{k}$ increases and that at $t_{k}=140 \mathrm{~s}$ the frequency responses of the reduced order model and the system match over a wide range of frequencies.

\section{CONCLUSION}

An algorithm for the estimation of the moments of linear SISO systems and linear time-delay SISO systems from input/output data has been given. The computational complexity of the algorithm has been discussed and a "fast" recursive least-square algorithm has been given. It has been proved that the estimates of the moments converge to the actual moments of the system. The estimate has been exploited to 

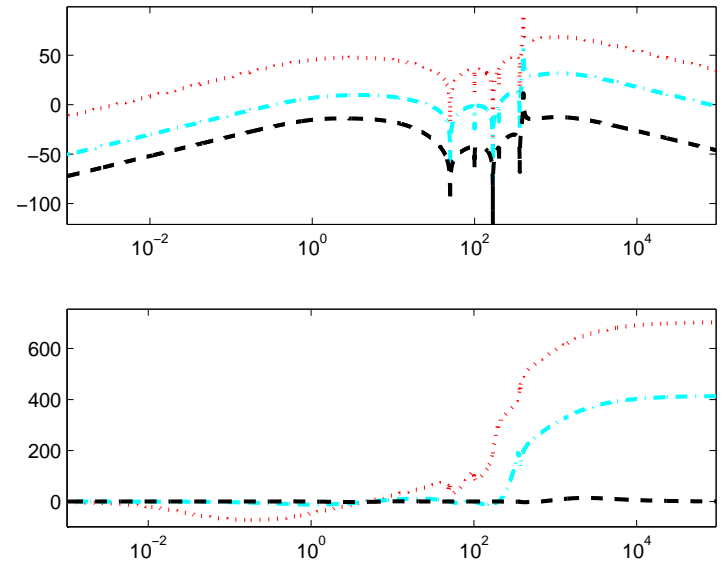

Fig. 2. Absolute errors between the Bode plot of the system to be reduced and the Bode plot of the reduced order model at $t_{k}=90 \mathrm{~s}$ (dotted line), of the reduced order model at $t_{k}=110 s$ (dash-dotted line) and of the reduced order model at $t_{k}=140 \mathrm{~s}$ (dashed line).

construct reduced order models which asymptotically match the moments of the system to be reduced. The problem of enforcing additional properties on the reduced order models has been discussed and solved. A benchmark system of order $n=1006$ has been reduced to illustrate the use of the algorithm. Future research will address the problem of extending the algorithm to nonlinear systems and to test it on specific applications.

\section{REFERENCES}

[1] K. J. Åström and P. R. Kumar, "Control: A perspective," Automatica, vol. 50, no. 1, pp. 3-43, 2014.

[2] V. M. Adamjan, D. Z. Arov, and M. G. Krein, "Analytic properties of Schmidt pairs for a Hankel operator and the generalized Schur-Takagi problem," Mathematics of the USSR Sbornik, vol. 15, pp. 31-73, 1971.

[3] K. Glover, "All optimal Hankel-norm approximations of linear multivariable systems and their $\mathrm{L}^{\infty}$-error bounds," International Journal of Control, vol. 39, pp. 1115-1193, 1984.

[4] M. G. Safonov, R. Y. Chiang, and D. J. N. Limebeer, "Optimal Hankel model reduction for nonminimal systems," IEEE Transactions on Automatic Control, vol. 35, no. 4, pp. 496-502, 1990.

[5] B. C. Moore, "Principal component analysis in linear systems: Controllability, observability, and model reduction," IEEE Transactions on Automatic Control, vol. 26, no. 1, pp. 17-32, 1981.

[6] D. G. Meyer, "Fractional balanced reduction: model reduction via a fractional representation," IEEE Transactions on Automatic Control, vol. 35, no. 12, pp. 1341-1345, 1990.

[7] W. S. Gray and J. Mesko, "General input balancing and model reduction for linear and nonlinear systems," in European Control Conference, Brussels, Belgium, 1997.

[8] S. Lall and C. Beck, "Error bounds for balanced model reduction of linear time-varying systems," IEEE Transactions on Automatic Control, vol. 48, no. 6, pp. 946-956, 2003.

[9] H. Kimura, "Positive partial realization of covariance sequences," Modeling, Identification and Robust Control, pp. 499-513, 1986.

[10] C. I. Byrnes, A. Lindquist, S. V. Gusev, and A. S. Matveev, "A complete parameterization of all positive rational extensions of a covariance sequence," IEEE Transactions on Automatic Control, vol. 40, pp. 1841-1857, 1995.

[11] T. T. Georgiou, "The interpolation problem with a degree constraint," IEEE Transactions on Automatic Control, vol. 44, pp. 631-635, 1999.

[12] A. C. Antoulas, J. A. Ball, J. Kang, and J. C. Willems, "On the solution of the minimal rational interpolation problem," Linear Algebra and Its Applications, Special Issue on Matrix Problems, vol. 137-138, pp. 511-573, 1990.
[13] C. I. Byrnes, A. Lindquist, and T. T. Georgiou, "A generalized entropy criterion for Nevanlinna-Pick interpolation with degree constraint," IEEE Transactions on Automatic Control, vol. 46, pp. 822-839, 2001.

[14] K. A. Gallivan, A. Vandendorpe, and P. Van Dooren, "Model reduction and the solution of Sylvester equations," in MTNS, Kyoto, 2006.

[15] C. A. Beattie and S. Gugercin, "Interpolation theory for structurepreserving model reduction," in 47th Conference on Decision and Control, Cancun, Mexico, 2008.

[16] A. Antoulas, Approximation of Large-Scale Dynamical Systems. Philadelphia, PA: SIAM Advances in Design and Control, 2005.

[17] A. Astolfi, "A new look at model reduction by moment matching for linear systems," in 46th IEEE Conference on Decision and Control, Dec 2007, pp. 4361-4366.

[18] _ - "Model reduction by moment matching for linear and nonlinear systems," IEEE Transactions on Automatic Control, vol. 55, no. 10, pp. 2321-2336, 2010.

[19] G. Scarciotti and A. Astolfi, "Model reduction by moment matching for linear time-delay systems," 19th IFAC World Congress, Cape Town, South Africa, August 24-29, 2014.

[20] — - "Model reduction by moment matching for nonlinear time-delay systems," in IEEE 53rd Annual Conference on Decision and Control, 2014.

[21] W. Dib, A. Astolfi, and R. Ortega, "Model reduction by moment matching for switched power converters," in Proceedings of the 48th IEEE Conference on Decision and Control, held jointly with the 28th Chinese Control Conference, Dec 2009, pp. 6555-6560.

[22] T. C. Ionescu and A. Astolfi, "Families of reduced order models that achieve nonlinear moment matching," in American Control Conference, Washington, DC, USA, June 17-19, 2013, pp. 5518-5523.

[23] T. C. Ionescu, A. Astolfi, and P. Colaneri, "Families of moment matching based, low order approximations for linear systems," Systems \& Control Letters, vol. 64, pp. 47-56, 2014.

[24] G. Scarciotti and A. Astolfi, "Characterization of the moments of a linear system driven by explicit signal generators," in Submitted to 2015 American Control Conference, 2015.

[25] Y. Jiang and Z. P. Jiang, "Computational adaptive optimal control for continuous-time linear systems with completely unknown dynamics," Automatica, vol. 48, no. 10, pp. 2699-2704, 2012.

[26] L. C. Baird, "Reinforcement learning in continuous time: advantage updating," vol. 4, pp. 2448-2453, Jun 1994.

[27] D. Vrabie, O. Pastravanu, M. Abu-Khalaf, and F. L. Lewis, "Adaptive optimal control for continuous-time linear systems based on policy iteration," Automatica, vol. 45, no. 2, pp. 477-484, 2009.

[28] E. N. Lorenz, Empirical Orthogonal Functions and Statistical Weather Prediction, ser. Scientific report 1, Statistical Forecasting Project. MIT, Department of Meteorology, 1956.

[29] C. W. Rowley, T. Colonius, and R. M. Murray, "Model reduction for compressible flows using POD and Galerkin projection," Physica D: Nonlinear Phenomena, vol. 189, no. 12, pp. 115-129, 2004.

[30] B. R. Noack, K. Afanasiev, M. Morzynski, G. Tadmor, and F. Thiele, "A hierarchy of low-dimensional models for the transient and posttransient cylinder wake," Journal of Fluid Mechanics, vol. 497, pp. 335-363, 122003.

[31] K. Willcox and J. Peraire, "Balanced model reduction via the proper orthogonal decomposition," AIAA Journal, vol. 40, no. 11, pp. 2323 2330, 2002.

[32] T. Penzl, "Algorithms for model reduction of large dynamical systems," Linear Algebra and its Applications, vol. 415, no. 23, pp. 322 -343 , 2006, special Issue on Order Reduction of Large-Scale Systems.

[33] K. Gallivan, A. Vandendorpe, and P. Van Dooren, "Sylvester equations and projection-based model reduction," Journal of Computational and Applied Mathematics, vol. 162, no. 1, pp. 213-229, 2004, proceedings of the International Conference on Linear Algebra and Arithmetic 2001.

[34] K. J. Åström and B. Wittenmark, Adaptive Control, ser. AddisonWesley series in electrical engineering : control engineering. AddisonWesley, 1995.

[35] T. N. E. Greville, "Some applications of the pseudoinverse of a matrix," SIAM Rev. 2, p. 1522, 1960.

[36] A. Ben-Israel and T. N. E. Greville, Generalized Inverses: Theory and Applications, ser. CMS Books in Mathematics. Springer, 2003.

[37] Q. Wang and L. Zhang, "Online updating the generalized inverse of centered matrices," 2011, pp. 1826-1827. 